\title{
Fabrication of Electrospun Polycaprolactone/Gelatin Composite Nanofibrous Scaffolds with Cellular Responses
}

\author{
Jaianand Kannaiyan, Saurabh Khare, Suriya Narayanan, Firdosh Mahuvawalla* \\ CelluGen Biotech Private Limited, Research and Development, Gurgaon, Haryana, India \\ Email address: \\ firdosh@cellugen.in (F. Mahuvawalla) \\ ${ }^{*}$ Corresponding author \\ To cite this article: \\ Jaianand Kannaiyan, Saurabh Khare, Suriya Narayanan, Firdosh Mahuvawalla. Fabrication of Electrospun Polycaprolactone/Gelatin \\ Composite Nanofibrous Scaffolds with Cellular Responses. American Journal of Nano Research and Applications. \\ Vol. 7, No. 2, 2019, pp. 11-20. doi: 10.11648/j.nano.20190702.11
}

Received: October 9, 2019; Accepted: November 4, 2019; Published: November 8, 2019

\begin{abstract}
Numerous wound care products have been investigated for skin tissue engineering. Factors which influence to skin substitute selection are condition of patient, wound depth, infection in wound. Other factors which also play role in deciding skin substitute are cost, its availability, ease of storage, required operative interventions. With perspective of Indian market, commercial available skin substitutes are either costly or their availability restricted to major cities only. A cost effective skin substitute is strongly needed to heal wounds with minimal scarring and maximum function. The aim of this study was to investigate the possibility of synthetic scaffold loaded with Wharton's jelly derived Mesenchymal stem cells and to access the role of scaffolds in proliferation and differentiation of MSCs in-vitro, in order to achieve for the healing of wound graft substitutes with improved biological properties. As a result, WJ-MSCs were isolated, harvested and seeded on the surface of the fabricated PCL/GE nanofibrous scaffold. The biological properties and growth of MSCs were studied for antiinflammation, cytotoxicity, cell proliferation, and SEM analysis indicated that the fabricated synthetic scaffold supported cells attachment, viability, and proliferation of cells. The characterization studies of nanofibers were studied for ATR-FTIR, XRD, TEM, viscosity, and degradation studies suggest that the nanofibrous scaffold loaded with stem cells could be an excellent tissue-engineered skin base for wound healing and skin regeneration.
\end{abstract}

Keywords: Electrospinning, Polycaprolactone (PCL), Gelatin (GE), Nanofibrous Scaffold, Mesenchymal Stem Cells (MSCs)

\section{Introduction}

Skin wounds continue to be a major public health concern worldwide resulting from surgical procedures, reduced circulations, mechanical trauma, burns, or aging [1]. Among all the injuries burn is a major global public health problem and also a main cause of morbidity [2, 3]. As stated by world health organisation (WHO) around 2,65,000 deaths occur each year due to fires alone globally, electrical burns, with more deaths from scalds and other forms of burns. In India, burn remains is the second largest group of injuries following road accidents with an estimated annual incidence of 6-7 million and nearly 1.4 lakh people die owing to severe burn while 2.4 lakh people suffer with disability [3]. Several natural skin substitutes such as allografts, xenografts and autografts have been widely used to restore wound defects.
However, these skin substitutes cannot achieve skin regeneration due to risk of infection, limited donor sites, slow healing and lead formation of scar $[4,5]$. In recent times, the rapid growth of nanotechnology has spurred the progression of nanofibrous scaffolds which has been found to play significant role in the management of skin-related disorders and deep burn [6].

Biomaterials scaffolds play an essential role in this technology by providing synthetic as well as suitable extra cellular matrix (ECM) surroundings for growing cells and drug delivery in critically injured skin [6]. There are numerous fabrication techniques including self-assembly, phase separation and electrospinning. Among all the techniques, Electrospinning is a fiber production technique which utilizes electric force for fabricating highly porous nanosize scaffolds from a wide variety of polymers 
comprises biopolymers such as gelatin, fibrinogen and collagen on a large scale [7]. The large surface area to volume ratio of nanofibers enhances the diffusion efficiency of nutrient and gaseous exchange [8]. Porosity, air permeability, and surface wettability of nanofibers are vital elements for tissue regeneration [9]. Moreover, electrospinning is capable to fabricate nanofibers with identical morphology and architectural features to the natural ECM in skin [10].

Fundamentally, natural and synthetic polymers are the principal scaffold materials utilize for tissue engineering applications. Among the synthetic polymers, PLGA (poly [lactic-co-glycolic acid]), PLLA (poly-L-lactic acid), PCL (Polycaprolactone) have been broadly used owing to their biocompatibility, biodegradability and appropriate mechanical properties [11-14]. In comparison to other synthetic polymers, polycaprolactone (PCL) is a bioresorbable and biocompatible polymer with outstanding mechanical properties, $[15,16]$ approved by food drug and administration (FDA) for usage in several biomedical applications. On the other hand, collagen, chitosan, gelatin, fibrinogen, chitin, and hyaluronic acid are commonly used natural polymer due to their superior biocompatibility [16, 17]. Among all natural polymers, gelatin has been generally used due to its low cost, easy availability and also contains the similar biological properties as collagen, a key element of the native ECM $[18,19]$. Therefore, the hybrid PCL/ Gelatin has gained considerable interests as excellent candidate for electrospinning fibrous scaffold for wound healing, layered dermal reconstitution and nerve tissue engineering [20,21] by taking the advantages of both synthetic as well as ECMlike natural polymers.

Similarly, Mesenchymal stem cells (MSCs) possess the ability to self-renew and express differentiation into various tissue-forming cell lineages, such as adipocytes, chondrocytes, osteoblasts, tenocytes and myocytes [22]. Wharton's jelly mesenchymal stem cells (WJ-MSCs) have stronger proliferation, differentiation, and migration abilities compared to bone marrow MSCs, and they are ideal seed cells for cell transplantation and tissue engineering for organ replacement. In addition, WJ-MSCs are good source for MSCs because they are globally available tissue and no ethical concern associated with their collection since they are usually discarded as human waste [23]. Therefore, mesenchymal stem cells isolated from Wharton's jelly become convenient alternative sources of MSCs for tissue repair and cell therapy in the field of regenerative medicine. In this study, we used PCL/Gelatin composite fibrous scaffolds seeded with mesenchymal stem cell for wound healing. This study investigates the potential and future prospects of synthetic scaffolds as skin substitutes fabricated using electrospinning for tissue repair and regeneration.

\section{Materials and Methods}

The study was conducted in R\&D, CelluGen Biotech, Gurgaon, India, after obtaining prior approval of the study protocol by the Institutional Committee for Stem Cell Research (IC-SCR).

\subsection{Material}

Poly ( $\varepsilon$-caprolactone) (PCL) was purchased from SigmaAldrich (UK). Gelatin (GE) in powder form was purchased from MP Biomedical (India) and its organic solvent 1, 1, 1, 3, 3, 3-hexafluoro-2-propanol (HFIP) (purity $\geq 99.0 \%$ ) was purchased from Alfa-Aesar (Product of Great Britain). All the other chemicals were of analytical reagent grade and used without further purification. The Umbilical cord tissue $(\mathrm{n}=$ 5) irrespective of the sex of baby was collected from fullterm births after caesarean section was obtained from the $\mathrm{C}^{-}$ section delivery process with donor consent form and institutional guidelines.

\subsection{Methods}

\subsubsection{Preparation of PCL/GE-Based Polymer Solution}

The $9 \%$ polymer blend solution of $\mathrm{PCL} / \mathrm{GE}$, which were $9 \%(\mathrm{w} / \mathrm{v})(\mathrm{PCL}=0.6 \mathrm{~g} ; \mathrm{GE}=0.3 \mathrm{~g})$, were prepared by dissolving PCL pellets and GE powder into $10 \mathrm{ml}$ of HFIP through stirring magnetically up to $12 \mathrm{~h}$ using a magnetic stirrer (REMI, India) until the mixture dissolve completely at room temperature.

\subsubsection{Fabrication of PCL/Gelatin-Based Electrospun Nanofibers}

An electrospinning unit (E-spin Nanotech, Super-ES-1, India) was used in the fabrication of PCL/GE -based nanofibers. The prepared polymer solution $(9 \%[\mathrm{w} / \mathrm{v}]$ PCL/GE-based) was transferred into a $5 \mathrm{ml}$ syringe with the constant flow rate of $0.5 \mathrm{ml} / \mathrm{h}$ using a syringe pump. Once the voltage at $14 \mathrm{kV}$ was applied to the needles of the syringe, a fluid jet was applied to the needles and accelerated towards a drum collector aluminium sheet. The needle was placed at a distance of $12.5 \mathrm{~cm}$ away from the aluminium collector. The polymer solution was evaporated and the charged polymer fibers were deposited on the collector in the form of nanofibers.

\subsection{Characterization of PCL/GE-Based Electrospun Nanofibers}

\subsubsection{Attenuated Total Reflectance (ATR): Fourier Transform Infrared Spectroscopy (FTIR)}

Chemical bonding of PCL/GE was determined by ATRFTIR. The functional groups present in the electrospun PCL gelatin nanofibers were analyzed using Fourier transforminfrared (FT-IR) spectrometer (Spectrum 100, Perkin Elmer, USA). The spectra were recorded between 4000 and 400 $\mathrm{cm}^{-1}$ with a resolution of $1 \mathrm{~cm}^{-1}$.

\subsubsection{X-ray Powder Diffraction (XRD)}

The electrospun samples were measured by analyzing the unique crystallography patterns for each of the material components obtained in X-ray diffraction (XRD) studies. A monochromatic $\mathrm{Cu}$ X-ray source $(40 \mathrm{kV})$ was utilized to 
characterize the effects of the nature of PCL over the range of $18-35^{\circ} 2 \theta$. A $30 \mathrm{~mm}$ disc with an average mat thickness of $140 \pm 11 \mu \mathrm{m}$ of each composition was punched out of fiber mats and then adhered to holders for XRD analysis.

\subsubsection{Viscosity}

Viscosity measurements of different PCL-GE concentration solution was carried out at different shear rates ranging from 0.1 to $500 \mathrm{~s}^{-1}$ at $25^{\circ} \mathrm{C}$. The spindle arrangement was parallel plate geometry with a gap of $100 \mu \mathrm{m}$ and $30 \mathrm{~mm}$ diameter plate.

\subsubsection{In-vitro Degradation Study}

The selected sample of nanofibers was cut into $1 \mathrm{~cm}^{2}$ pieces and immersed in phosphate buffered saline (PBS, $\mathrm{pH}$ $=7.4$ ) and incubated in-vitro at $37^{\circ} \mathrm{C}$ for different periods of time (day 1 and day 28). After each degradation period, the nanofibers were washed and subsequently dried in room temperature for 24 hours and all the samples were rinsed twice with distilled water to remove salts and then dried the weight of the dried samples were measured and the percentage weight loss was calculated using following formula:

$$
\text { Weight loss }(\%)=\frac{\text { Initial weight-final weight }}{\text { initial weight }} \times 100
$$

\subsubsection{Transmission Electron Microscopy of Core-Shell Structure}

Transmission electron microscope (TEM, FEI-TECNAI G2 20S-TWIN, FEI, Hillsboro, USA) was performed to ascertain the core-shell structure of nanofibers. The nanofibers with different combinations of PCL-SF were directly electrospun on the carbon coated grids of TEM and observed under $80 \mathrm{kV}$, low voltage was applied for nanofibers to avoid radiation damage.

\subsection{In-vitro Stem Cells Experiments and Characterization Studies}

\subsubsection{Stem Cells Culture}

The human umbilical cord was retrieved after caesarean section with informed consent. WJ-MSCs were isolated from umbilical cord via explant culture as described in the previous publication. Briefly, 35-40 jelly explants with size of $0.5 \mathrm{~mm}$ were transferred in tissue-culture-grade T-75 flask (Nunc, Denmark) which contained culture medium. After incubation at $37^{\circ} \mathrm{C}$ for $3-5$ days non-adherent cells were removed and fresh media was added. In this study, MSCs were cultured and expended in Dulbecco's modified Eagle medium-Nutrient mixture Ham's F-12 (1:1) with Glutamax (1X); $2.438 \mathrm{~g} / \mathrm{L}$ Sodium Bicarbonate; Sodium Pyruvate (DMEM/F12+; Gibco, USA) with 10\% PLTMax Human Platelet Lysate (SCM141, Merck) supplemented with 2 $\mathrm{ng} / \mathrm{mL}$ basic fibroblast growth factor (bFGF; Sigma-Aldrich, UK). Cells were maintained in a $5 \% \mathrm{CO}_{2}$ incubator (Thermo scientific, USA) at $37^{\circ} \mathrm{C}$ and the medium was renewed every five days. After reaching 70-80\% confluency cells were dissociated using TrypLE Express (Gibco, USA) and passaged 1:3 into fresh culture flasks.

\subsubsection{Characterization of Stem Cells}

All the experiments were performed with WJ-MSCs from passage 1-2. Thereafter, cells were characterized for surface antigen expression using flow cytometry analysis (FACSCalibur, BD Biosciences, USA) and data analysis was done using the CellQuest Prosoftware (BD Biosciences, USA) [24]. The cells were positive for the cell surface markers were analysed with CD90-FITC, CD73-APC, CD105-PE (BD Pharmingen, USA) and negative for CD45FITC, CD34-PE, CD79a-APC and HLA-DR BD Pharmingen, USA). The ability to differentiate into osteogenic, chondrogenic, and adipogenic lineages using specific culture media were studied as per previous studies $[24,25]$. The experiments are conducted using the guidelines approved by Inter-National Society for Cellular Therapy (ISCT) to define human MSCs [26].

\subsection{Characterization of the MSCs Seeded Scaffolds}

\subsubsection{Cytotoxicity Evaluation of Scaffolds}

The cytotoxicity of MSCs on synthetic scaffold was studied via MTT (3-(4, 5-Dimethylthiazol-2-yl)-2, 5diphenyltetrazolium bromide) assay after 48 hours [27, 28]. The method of analysis employed using standard techniques was studied in detail in the previous published publication [29].

\subsubsection{Cell Attachment and Cell Proliferation}

Cell proliferation was analysed by using the Alamar blue (AB) assay [30]. The cell scaffold constructs were removed from the culture plates at days 1, 3, 5, 7, 14 and 21, using standard conventional protocols were studied in detail in the earlier published methodology [29].

\subsubsection{Anti-Inflammation Study}

The experiment was carried out with minor modification Gnana et al. 2011 [31]. The standard drug and test samples (PCL/Gelatin nano fibers) were diluted with phosphate buffer (0.2 M, pH 7.4). The different concentrations of standard drug and test samples was mixed with $1 \mathrm{ml}$ of $1 \mathrm{mM}$ albumin solution in phosphate buffer and incubated at $37^{\circ} \mathrm{C}$ in incubator for $15 \mathrm{~min}$. Denaturation was induced by keeping the reaction mixture at $60^{\circ} \mathrm{C}$ in water bath for $15 \mathrm{~min}$. After cooling, the turbidity was measured at $660 \mathrm{~nm}$. Percentage of inhibition of denaturation was calculated from control where no drug was added. The diclofenac sodium was used as standard drug. The percentage inhibition of denaturation was calculated by using following formula.

$\%$ of Inhibition $=[(\mathrm{OD}$ of test

$$
\text { - OD of control)/OD of test] x } 100
$$

\subsubsection{Cell Attachment and Morphology}

Cell attachment and morphology of cells seeded on the synthetic scaffolds were studied via scanning electron microscopy (SEM). Samples were collected at different time intervals, at the end of particular incubation time, cell- 
scaffold constructs were washed twice with PBS and kept in $2.5 \%$ glutaraldehyde (TCI, India) solution for 2 hours at room temperature and maintained at $-80^{\circ} \mathrm{C}$ as per the published protocol [29].

\subsection{Statistical Analysis}

All statistical analyses were performed using GraphPad Prism Software (La Jolla, CA, USA). Results were expressed as mean \pm standard deviation (SD) for illustration. The statistical significance of metabolic activity and proliferation potential among harvested cells was determined using twoway ANOVA. $\mathrm{P}<0.0001$ was considered statistically significant.

\section{Result and Discussion}

Engineering skin substitute by tissue engineering approach is based on creation of three dimensional scaffold analogue to extra cellular matrix which guides cell adhesion and proliferation. The interest in stem cells for tissue engineering and regenerative medicine has been increased because of properties possessed by stem cells such as proliferation potential, multi lineage differentiation potential and capacity to modulate immune phenotype $[32,33]$. A major constrain to stem cell therapeutic to clinical application is the supply of adequate number of cells [34]. 3D scaffolds provide a better resemblance of natural environment which can hardly be represented by $2 \mathrm{D}$ plastic substrate [35]. There are various techniques available for scaffold fabrication such as phase separation, freeze drying, electrospinning, solvent casting, rapid prototyping, 3D printing etc.

Among, the electrospinning is a technique used widely for fabricating fibers with diameters in the nanoscale $(<1000 \mathrm{~nm})$ or microscale $(>1 \mu \mathrm{m})$ range $[7,8,36]$. In the electrospinning process, a syringe pump, a high voltage source, and a collector are needed. Firstly, a very high voltage is applied to a capillary tube filled with polymer solution or melt, which is held at the tip of the capillary via surface tension. Secondly, a mutual charge repulsion caused by application of an electrical field is induced within the polymer solution or melt, which directly opposes the surface tension of the polymer solution. When the intensity of the electrical field is increased, the charge repulsion will overcome the surface tension to form a jet. Finally, the ejected polymer solution and melt repel each other and the solvent evaporates to form fibers as the jet travels to the collector. Fibers ranging from nanometers to micrometers in size can be formed by regulating parameters including intrinsic solution properties, e.g., viscosity, conductivity, and surface tension, and operational conditions, including the hydrostatic pressure in the capillary tube, strength of the electrical field applied, and distance between the tip and collector.

The present study aims to develop PCL/GE scaffold loaded with cells and evaluate its biological efficacy. The cell engraftment with biomaterial can further enhance the potential of scaffolds in repair or regenerate the tissue defects. The development of cell loaded scaffold may enable complete, accelerated wound regeneration. The following outcomes of the study revealed the positive desired properties of the fabricated synthetic composite.

\subsection{Surface Morphology Analysis}

Random nanofibers were fabricated through electrospinning. Defect-free random nanofibers were achieved by the optimized the solution and process parameter includes solvent ratio, polymer concentration, flow rate, applied potential, tip-to-target distance and needle size. The electro spinning process parameters for PCL-gelatin blend system were optimized and are PCL-gelatin (6:3) at 9\% (w/v) concentration when electrospun at $14 \mathrm{kV}$ applied potential, $0.5 \mathrm{~mL} / \mathrm{h}$ flow rate, $12.5 \mathrm{~cm}$ of tip-to target distance and $16 \mathrm{G}$ needle size formed defect-free nanofibers and the average nanofiber diameter was $228 \pm 30 \mathrm{~nm}$.

\subsection{Attenuated Total Reflectance (ATR): Fourier Transform Infrared Spectroscopy (FTIR)}

The characteristic groups present in the PCL-gelatin nanofibers were confirmed using FTIR spectroscopy. FTIR figure shows the FTIR spectra of PCL-gelatin nanofibrous scaffolds. The PCL nanofiber scaffolds showed characteristic bands at $2939.52 \mathrm{~cm}^{-1}$ (asymmetric CH2 stretching), 2862.36 $\mathrm{cm}^{-1}$ (symmetric $\mathrm{CH} 2$ stretching), $1724.36 \mathrm{~cm}^{-1}$ (ester carbonyl stretching), $1294.24 \mathrm{~cm}^{-1}$ (C-O stretching) and $1240.23 \mathrm{~cm}^{-1}$ (asymmetric COC stretching). The PCL gelatin nanofibrous scaffold exhibited characteristic bands at $1649.14 \mathrm{~cm}^{-1}$ (amide I), $1544.98 \mathrm{~cm}^{-1}$ (amide II), $1456.26 \mathrm{~cm}^{-}$ ${ }^{1}$ (amide III), and $1365.60 \mathrm{~cm}^{-1}$ (amide IV) along with characteristic bands due to the PCL. FTIR spectrum of PCLgelatin nanofiber showed characteristic bands of both constituent polymers confirming formation of the blend (Figure 1a). The presence of an amide group confirms the presence of GE in nanofibers after polymer blending and electrospinning. Amide groups in gelatin are able to form hydrogen bonds with water molecules. Thus gelatin has the ability to increase the hydrophilicity of PCL based nanofibers. The samples a to f tested are Sample (a): PCL gelatin optimized polymer solution and process parameters on PCL-gelatin nanofiber scaffold, Sample 1(b) to 1(f) are scaffold exposed with D. $\mathrm{H}_{2} \mathrm{O}, \mathrm{PBS}, \mathrm{EtoH}, \mathrm{CHCl}_{3}$, and DCM respectively for analyze the degradation of nanofibers in different solvents. All the exposed samples are observed almost similar type of characteristic bands of FTIR spectrum (Figure 1).

\section{3. $X R D$}

X-rays scatter off of electrons, in a process of absorption and re-admission. Diffraction is the accumulative result of the x-ray scattering of a group of electrons that are spaced in an orderly array. For an incident X-ray photon of monochromatic wavelength $\lambda$, coherent waves are produced from the sample at an angle of $\theta(2-\theta$ with respect to the incident $\mathrm{x}$-ray beam) if the electron groups interact with the $\mathrm{x}$-ray beam and are spaced at a repeat distance $\mathrm{d}$. The 
interaction is described by Bragg's law: $\mathrm{n} \lambda=2 \mathrm{~d} \sin \theta$. The intensity of the scattered $\mathrm{x}$-ray is proportional to the number of electrons that the $\mathrm{x}$-ray is scattered from. Figure 2 shows the XRD patterns for PCL, The PCL exhibits strong peaks at 25.15 and $26.75^{\circ}$ in $2 \Theta$. However, small peaks seen at 21.25
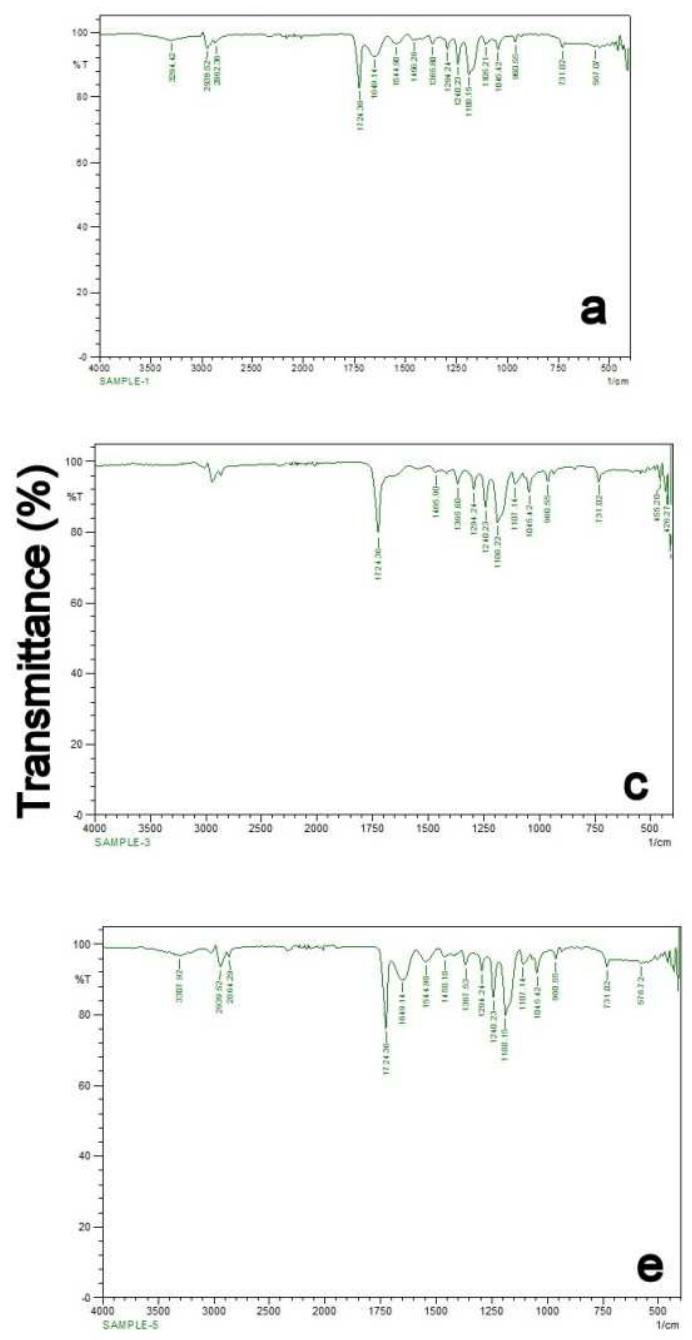

\section{Wave number $(\mathrm{cm}-1)$}

Figure 1. Chemical bonding analysis by ATR-FTIR: The samples a to f tested are Sample (a): PCL gelatin optimized polymer solution and process parameters on PCL-gelatin nanofiber scaffold, Sample (b) to (f) are Scaffold exposed with D. $\mathrm{H}_{2} \mathrm{O}, \mathrm{PBS}, \mathrm{EtoH}, \mathrm{CHCl}_{3}$, and DCM respectively.

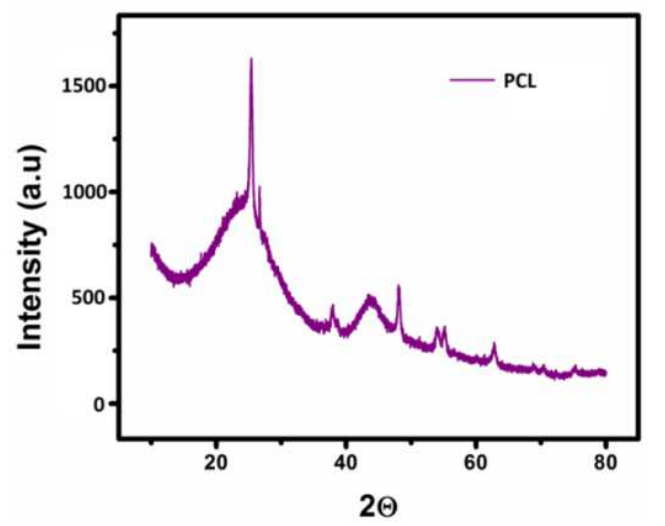

Figure 2. XRD Patterns for the electrospun fiber: PCL shows high crystallinity with peaks at 25.15 and $26.75^{\circ}$ in $2 \Theta$.

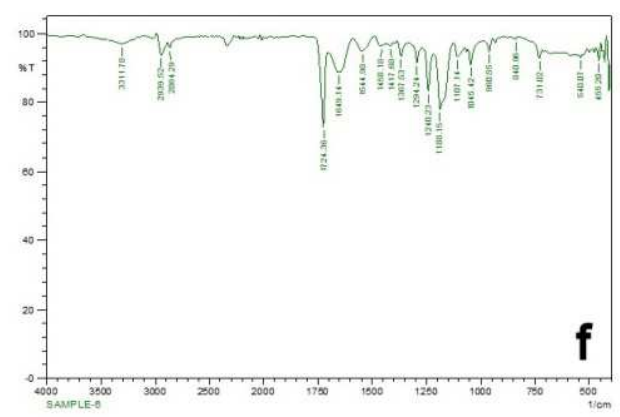

and $23.75^{\circ}$ in $2 \Theta$ represent a PCL component of the composite suggesting a blended material. XRD analysis adds additional confirmation that electrospun composite scaffolds were produced.
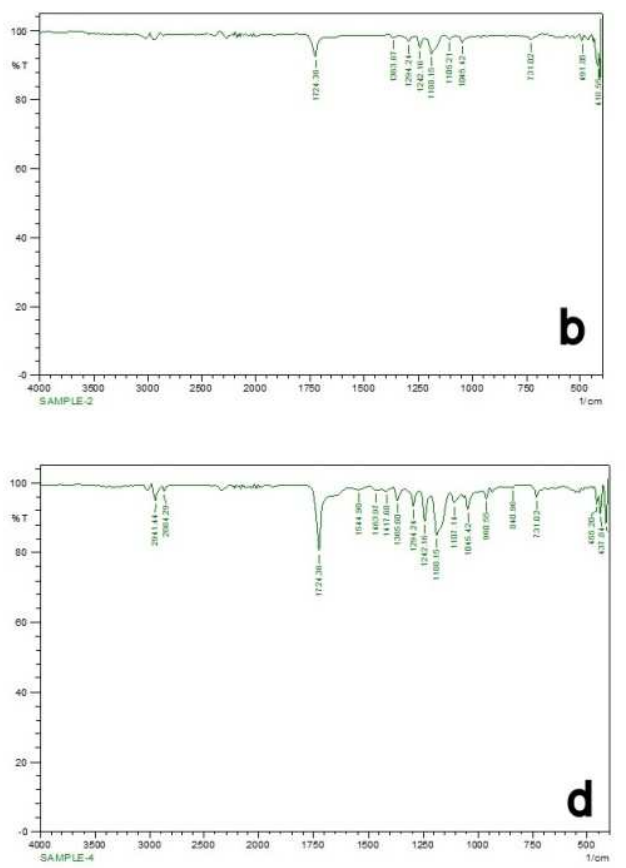

\subsection{Viscosity}

Viscosity of the electrospinning solution plays a dominant role in determining the nanofiber formation ability, dimension and texture of the fibers. All the emulsion combinations exhibited non-Newtonian shear thinning property under the application of shear rate. It has been reported [37] that sufficient chain entanglement was necessary among the polymers for initiation of uninterrupted electrospinning yielding fibers in diameter submicron range. Viscosity with both very low and high values might result in beaded nanofibers; an optimum value of viscosity in the range of 0.1 to $2 \mathrm{~Pa}-\mathrm{s}$ is preferred for spinning [35]. In the conducted study with $9 \%$ PCL/GE, it was observed that PCL solution displayed higher viscosity and the stability of the 
nanofibers produced was intact but had thicker fiber diameters. The viscosity of the final $9 \% \mathrm{PCL} / \mathrm{GE}$ was $0.1 \mathrm{~Pa}-$ $\mathrm{S}$ which resulted in breakage of solution into droplets giving rise to multiple beaded fibers.

\subsection{In-vitro Degradation Study}

Degradation of PCL - gelatin nano fibers were studied for 4 weeks, the synthetic scaffolds in the study were cut into square shaped samples with dimension of $10 \mathrm{~mm} \times 10 \mathrm{~mm}$. Samples were kept in PBS solution at $37^{\circ} \mathrm{C}$ in a shaking water bath and the PBS was changed every alternate day. The PCL- gelatine nano fibers were removed after, 7, 14, 21 and 28 days. In figure $3 \mathrm{~A}$ shows the morphology degradation of PCL-gelatine nanofibrous from day 0 to day 28 , and observed that $>50 \%$ of scaffold was degraded after 28 days. The figure 3B shows weight loss percentage of PCL- gelatine nanofibrous scaffold was calculated for synthetic membrane $(\mathrm{n}=5)$ for $7,14,21$ and 28 days correspondingly expresses the mean of weight loss percentage of PCL-gelatine nanofibrous scaffold after in-vitro degradation on 7, 14, 21, and 28 days.
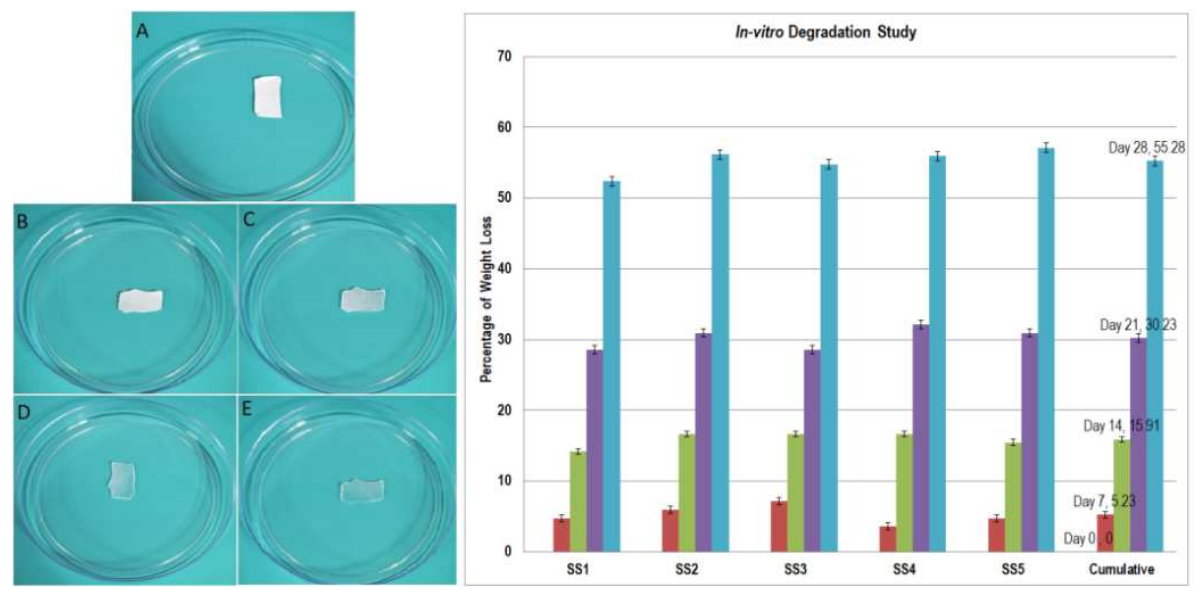

Figure 3. Morphology of degraded nanofibers: (A) In-vitro morphology degradation of PCL-Gelatin nano fibres (A to E) on 7, 14, 21, and 28 days correspondingly, (B): Weight loss percentage of PCL/GE nanofibrous was calculated for synthetic scaffold ( $n=5)(* S S)$ after in-vitro degradation on 7, 14,21 , and 28 days respectively.

\subsection{Transmission Electron Microscopy of Core-Shell Structure}

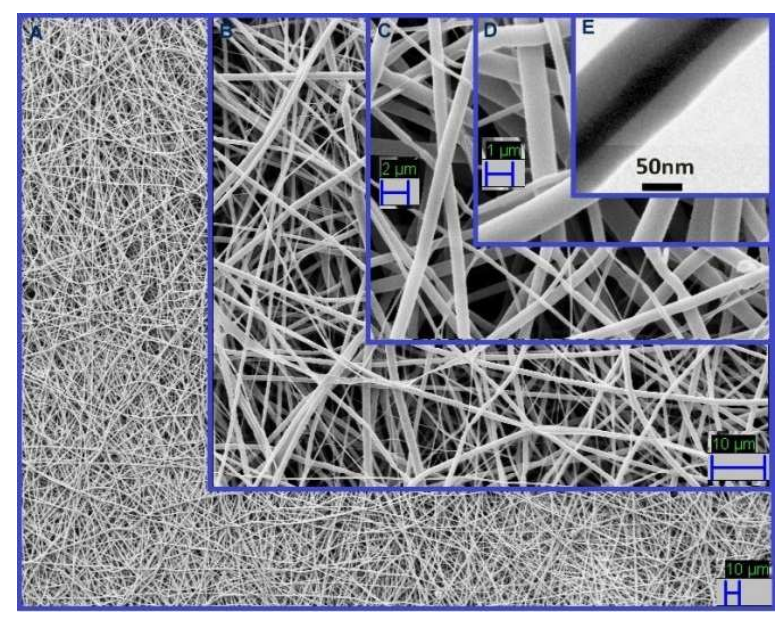

Figure 4. The PCL/GE nanofibers exhibited distinct core-shell structure with fiber diameter was observed by TEM in Figure E, Figure A to D is the morphology under SEM of nano fiber scaffold with magnification of $500 X$, $2 K X, 5 K X$, and $10 K X$ respectively.

Transmission electron microscopy (TEM) was performed to ascertain the core-shell structure of the electrospun nanofibers. As the polymer jet is drawn more towards the collector plate under high electrical voltage, the aqueous phase is drawn inwards in the individual fibers giving rise to core-shell structure. The nanofibers with the optimized combinations at $9 \%(\mathrm{w} / \mathrm{v}) \mathrm{PCL}$ - Gelatin (6:3) polymer concentration, $14 \mathrm{kV}$ applied potential, $0.5 \mathrm{~mL} / \mathrm{hr}$ flow rate, at a tip-to-target distance of $12.5 \mathrm{~cm}$ were directly electrospun on the carbon coated grids of TEM and observed under $80 \mathrm{kV}$, low voltage was analyzed. The PCL/GE nanofibers exhibited distinct core-shell structure with fiber diameter was observed in the average was found to be $228 \pm$ $30 \mathrm{~nm}$ under the optimized conditions (Figure 4).

\subsection{Cytotoxicity Evaluation}

The cytotoxicity of synthetic scaffold seeded with WJMSCs ( $n=5)$ was confirmed by quantitative analysis using the MTT assay. The absorbance values of test samples (TS1 and TS2) after exposure to the medium for 48 hours were corresponding to the number of metabolically active cells. The absorbance values of TS1 and TS2 showed good viability and continuous metabolic activity of the MSCs on scaffold than the positive control (PC) and known cells control (KC1-KC4). Thus the results (Figure 5) showed that the metabolic activity of WJ-MSCs on fabricated scaffold was not suppressed in the medium and from the computable scores, it was determined that the extracts of the fabricated biological scaffolds proved no cytotoxic reactivity in this experiment. The statistical analysis used for determine the cytotoxicity effect by One-way ANOVA, $\mathrm{P}$ value $\mathrm{P}<0.0001$, with means significant difference $(\mathrm{P}<0.05)$, and the data represent cumulative mean $\pm \mathrm{SD}$ is statistically significant. 


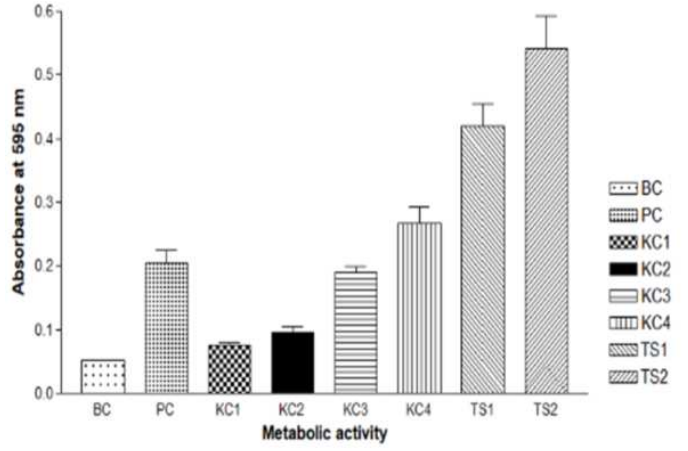

Figure 5. Data represent cumulative mean $\pm S D$ for determination of cytotoxicity of synthetic scaffolds $(n=5)$ : (BC): Blank Control - Culture medium without scaffold and Cells, (PC): Positive Control - Synthetic scaffold without cells, (KCl to KC4): Known Control Sample - 25000, 50000, 75000 and 100000 MSCs seeded without scaffold respectively, (TS1 and TS2): Test sample - 50000 and 100000 cells seeded on synthetic scaffold respectively. (Absorbance at $595 \mathrm{~nm}$ ).

\subsection{Cell Attachment and Cell Proliferation}

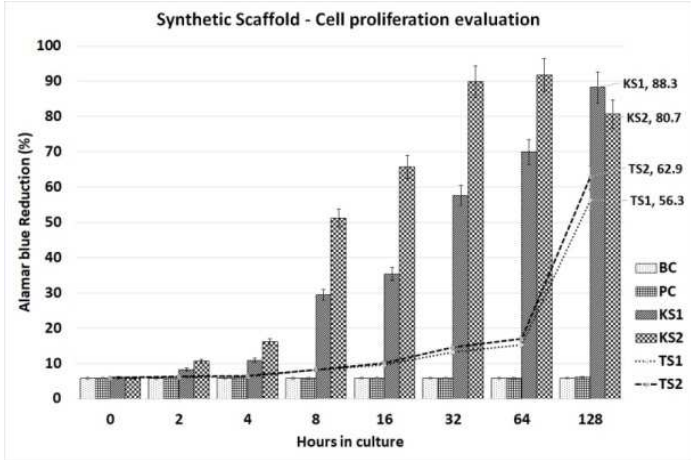

Figure 6. A Standard graph of percentage AB reduction versus logarithm of cell growth. Test parameter: Absorbance values at wavelengths of $540 \mathrm{~nm}$ and 630nm Vs Hours in culture. Test sample (TS1 and TS2): 50000 and 100000 cells seeded on synthetic scaffold, and known sample control (KS1 and KS2): 50000 and 100000 MSCs seeded without scaffold respectively. Whereas blank control (BC): culture medium without scaffold and cells, and Positive control (PC): Synthetic scaffold without cells accordingly.

The results confirms the percentage reduction of $\mathrm{AB}$ with different incubation hours and at different initial cell densities and including the standard graph of percentage (\%) $\mathrm{AB}$ reduction versus logarithm of cell growth (Figure 6). The higher $\mathrm{AB}$ reduction was observed in the test culture TS2 compared with TS1 over the whole culture period. In the culture, cell proliferation was gradually increased with culture time over the first 3 days and the metabolic activity of the cells (KS1 and KS2) growing in the medium seems to slow-down by day 5 , suggesting that the surfaces were advancing into maximum confluence. Similarly, AB reduction percentage of TS1 and TS2 was started increased after 64 hours were showed that the constant cells growth rate and quantified proliferation of MSCs on the scaffold.

\subsection{Anti-Inflammation Study}

The anti-inflammatory activity of PCL/Gelatin nano fibers was studied and the anti-inflammatory mechanisms of PCL/Gelatin nano fibers seeded with mesenchymal stem cells secreted cytokines might be released, resulting the decrease in the level of inflammation. The results were obtained from the Control: Diclofenac sodium and changes in the turbidity of the PCL/Gelatin nano fibers seeded with Mesenchymal Stem Cells for anti-inflammatory activity was measured at $660 \mathrm{~nm}$ were tabulated in the below Table 1 and the percentage of inhibition is demonstrated in figure 7 . Denaturation of proteins is a known cause of the inflammation. As part of this investigation on the mechanism of the anti-inflammation activity, ability of synthetic scaffold seeded with MSCs to inhibit the protein denaturation was studied. Percentage inhibition of PCL/Gelatin nano fibers seeded with Mesenchymal Stem Cells was calculated from $50 \mu \mathrm{g} / \mathrm{ml}$ to $1600 \mu \mathrm{g} / \mathrm{ml}$ concentration. It is observed that $19.08 \%$ at $50 \mu \mathrm{g} / \mathrm{ml}, \quad 33.5 \%$ at $100 \mu \mathrm{g} / \mathrm{ml}, \quad 43.67 \%$ at $200 \mu \mathrm{g} / \mathrm{ml}, 50.85 \%$ at $400 \mu \mathrm{g} / \mathrm{ml}, 63.28 \%$ at $800 \mu \mathrm{g} / \mathrm{ml}$ and the maximum inhibition of $75 \%$ was observed at $1600 \mu \mathrm{g} / \mathrm{ml}$ respectively (Figure 8 ). No changes in the turbidity were recorded in the untreated control. This study result reveals that the effective inhibition of protein denaturation proved the potential anti-inflammatory activity of PCL/Gelatin nano fibers seeded with Mesenchymal Stem Cells.

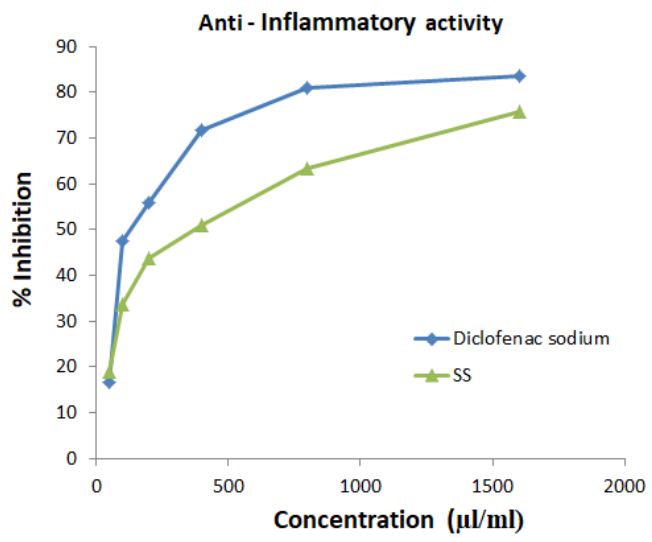

Figure 7. Percentage inhibition of sythetic scaffold seeded with mesenchymal stem cells with variation in concentration of diluted test samples.

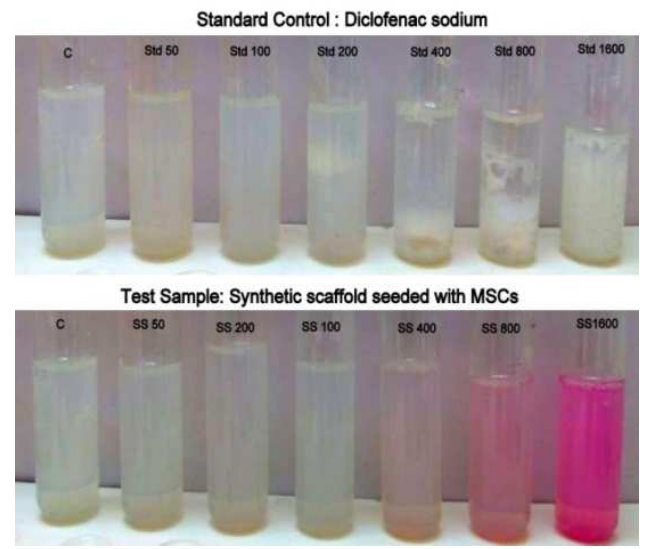

Figure 8. The anti-inflammatory activity of PCL/Gelatin nano fibers: The anti-inflammatory activity of $\mathrm{PCL} /$ Gelatin nano fibers. (A) Control: Diclofenac sodium was used as standard drug (B) Changes in the turbitity of the PCL/Gelatin nano fibers seeded with Mesenchymal Stem Cells for protein denaturation test. 
Table 1. Anti-inflammatory activity.

\begin{tabular}{|c|c|c|c|c|c|c|c|c|}
\hline \multirow[b]{2}{*}{ Conc.( $(\mu \mathrm{g})$} & \multicolumn{3}{|c|}{ OD at $660 \mathrm{~nm}$} & \multicolumn{3}{|c|}{$\%$ of Inhibition } & \multirow[b]{2}{*}{ mean } & \multirow[b]{2}{*}{ sd } \\
\hline & Singlet & Duplicate & Triplicate & Singlet & Duplicate & Triplicate & & \\
\hline 50 & 0.299 & 0.302 & 0.305 & 18.2832 & 19.0949 & 19.8907 & 19.0896 & 0.80379 \\
\hline 100 & 0.361 & 0.368 & 0.375 & 32.3176 & 33.6051 & 34.8444 & 33.5891 & 1.26348 \\
\hline 200 & 0.421 & 0.439 & 0.442 & 41.9636 & 44.3432 & 44.721 & 43.6759 & 1.49491 \\
\hline 400 & 0.489 & 0.495 & 0.508 & 50.0341 & 50.6397 & 51.9029 & 50.8589 & 0.95348 \\
\hline 800 & 0.663 & 0.657 & 0.677 & 63.1473 & 62.8108 & 63.9094 & 63.2892 & 0.56289 \\
\hline 1600 & 0.991 & 1.011 & 1.017 & 75.3448 & 75.8325 & 75.9751 & 75.7175 & 0.33054 \\
\hline
\end{tabular}

\subsection{Cell Attachment and Morphology}

The morphology and attachment of cells seeded on the synthetic scaffolds were examined via scanning electron microscopy. Figure 9 shows the SEM micrographs of the MSCs cultured on the surface of PCL/Gelatin membranes for 3 and 5 days. Most of the MSCs were well flattened and favorably spread across all sample surfaces for both third and fifth days, indicating good interaction and integration of MSCs with the surrounding fibers. It is observed at third-day that MSCs adhesion as well as the highest numbers of cells adhered on the nanofibrous surfaces. Similar trend has been found at fifth-day culture, where the PCL/GE sample again showed the best MSC adhesion and had most cells adhered to its surface (figure 9). It is also noted that the fibrous structure appeared quite stable after fifth-day incubation in the culture medium based on the SEM observations, and no indication of the preferential dissolving of the gelatin was found according to the attenuated total reflectance Fourier transform infrared spectroscopy measurements.

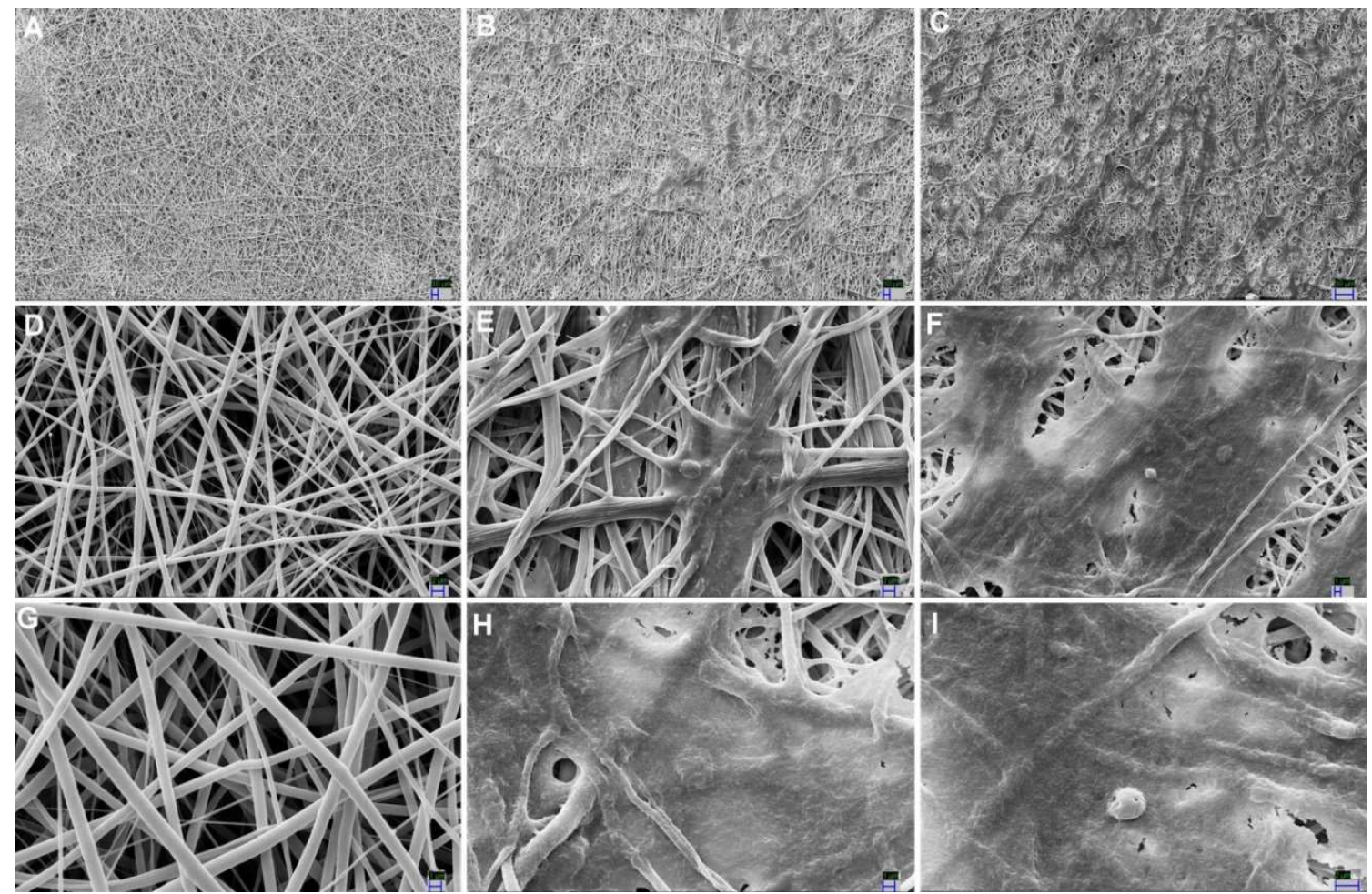

Figure 9. Synthetic nano fiber scaffold under SEM Magnification: (A, D, G) Control: Morphology of the PCL/Gelatin electrospun fibers (Magnification 500X, $5000 X$ and 10,000X respectively), (B, E, H): Morphology of MSCs cultured for 3 days on the PCL/Gelatin electrospun nanofiber scaffold appreance and MSCs are adhered onto synthetic scaffold (Mag: 500X, 5000X and 10,000X respectively), and (C, F, I): Morphology of MSCs cultured for 5 days on the PCL/Gelatin electrospun nanofiber scaffold appreance and MSCs are adhered onto synthetic scaffold (Mag: 500X, 5000X and 10,000X correspondingly).

Taken altogether, The study proved that the PCL/Gelatin sample not only indicated the uppermost tensile strength and elongation rate, also the finest MSC responses in terms of attachment, spreading, viability, proliferation and cytoskeleton organization. The result suggested that the mechanical properties of the nanofibrous scaffold could be a significant part of the cellular microenvironment and play a vital role in affecting the cellular responses.

\section{Conclusion}

Cell-based tissue engineering provides a huge hope for regenerative therapy, but it has been limited so far due to the insufficient number of cells obtained from donors and the lack of effective ways to deliver them to target sites [34, 35]. To overcome the challenge, a special processing technique is 
required to ensure consistent quality and promising option for wound healing matrices to mimic extracellular matrix of skin, nanoporous nature to aid in the transport of oxygen to the wound while keeping bacteria out and bioactive factors can be incorporated into the nanofibres to further promote healing. In this study, we have developed the fabricated skin substitute consists of electrospun nanofibrous seeded with mesenchymal stem cells composite system, with focused on the advantages of artificial scaffold approach over biologic scaffold, in terms of the fabricated raw chemical materials; is biodegradable, can be fabricated in large size according to clinical demand, not have any risk of disease transmission in comparison to natural derived material; easy to use and available 'off-the-shelf'. The overall results suggested that the nanofibrous network mechanical properties could be an important part of the cellular microenvironment and play an important role in influencing cellular responses. In addition to proving the finest MSC responses in terms of attachment, proliferation, and matrix formation, the PCL/ Gelatin nanofibrous also had the highest tensile strength and prolonged rate. Thus, it's clearly evidenced that electrospun nanofibers allows the design and fabrication of biomimetic scaffolds that offer an architecture, a fabulous potential uses for wound healing, also can deliver the stem cells at target site to makes it a promising candidate for expansion and transplantation of stem cells in large numbers at a target defect site.

\section{Funding Sources and Acknowledgments}

This study was supported by a Small Business Innovation Research Initiative (SBIRI) funded by the Biotechnology Industry Research Assistance Council, India (BT/SBIRI1436/33/17). We also wish to thank Lalit Jaiswal and Kangan Jaiswal of CelluGen Biotech Pvt. Ltd. Gurgaon for the support.

\section{Consent for Publication}

J. K performed experiments, analysed the data, and wrote the manuscript. S. K. performed experiments. S. N. and F. M. interpreted the data. All read and finalized the manuscript.

\section{Disclosure of Potential Conflict of Interest}

The authors declare that they have no competing interests.

\section{References}

[1] Allgower M, Schoenberger GA, Sparkes BG. "Burning the largest immune organ,” Burns 1995; 21: S7-S47.

[2] Forjuoh SN. "Burns in low- and middle-income countries: a review of available literature on descriptive epidemiology, risk factors, treatment, and prevention," Burns 2006; 32: 529.
[3] Peck MD, Kruger GE, van der Merwe AE, Godakumbura W, Ahuja RB. "Burns and fires from non-electric domestic appliances in low and middle income countries Part I. The scope of the problem," Burns 2008; 34: 303.

[4] Price R, Anthony E, Myers S, Navsaria H. "Tissue engineering for skin transplantation," Tissue Engineering, Burlington, Academic Press 2008; 507-532.

[5] Clark RAF, Ghosh K, Tonnesen MG. "Tissue engineering for cutaneous wounds," J Invest Dermatol 2007; 127: 1018-1029.

[6] Chong LH, Lim MM, and Sultana N, "Polycaprolactone (PCL)/gelatin (Ge)-based electrospun nanofibers for tissue engineering and drug delivery application," Applied Mechanics and Materials 2014; 554: 57-61.

[7] Bhardwaj N and Kundu SC. "Electrospinning: a fascinating fiber fabrication technique," Biotechnology Advances 2010; 28 (3): 325-347.

[8] Sill TJ and Von Recum HA. "Electrospinning: applications in drug delivery and tissue engineering," Biomaterials 2008; 29 (13): 1989-2006.

[9] Liu X, Lin T, Fang J, Yao G, Zhao H, Dodson M, Wang X. "In vivo wound healing and antibacterial performances of electrospun nanofibre membranes," Journal of Biomedical Materials Research 2010; 94 (2): 499-508.

[10] Chong EJ, Phan TT, Lim IJ, Zhang YZ, Bay BH, Ramakrishna S, Lim C T. "Evaluation of electrospun PCL/gelatin nanofibrous scaffold for wound healing and layered dermal reconstitution," Acta Biomaterialia 2007; 3 (3): 321-330.

[11] Okada M. "Chemical syntheses of biodegradable polymers," Prog. Polym. Sci 2002; 27: 87-133.

[12] Ranjbar-Mohammadi M, Zamani M, Prabhakaran M, Bahrami SH, Ramakrishna S. "Electrospinning of PLGA/gum tragacanth nanofibers containing tetracycline hydrochloride for periodontal regeneration,” Mater. Sci. Eng. C. 2016; 58: 521-531.

[13] Zhou Q, Xie J, Bao M, Yuan H, Zhaoyang Y, Loua XB and Zhang Y. "Engineering aligned electrospun PLLA microfibers with nanoporous surface nanotopography for modulating the responses of vascular smooth muscle cells," J. Mater. Chem. B. $2015 ; 3$ : 4439-4450.

[14] Lee K, Kim H, Khil M, Ra Y. "Characterization of nanostructured poly (E-caprolactone) nonwoven mats via electrospinning," Polymer 2003; 44: 1287-1294.

[15] She H, Xiao X, Liu R. "Preparation and characterization of polycaprolactone-chitosan composites for tissue engineering applications," J. Mater. Sci. 2007; 42: 8113-8119.

[16] Jaianand K, Hemlata C, Palaniyandi M, Rajangam B, Suriya S, Anubhav P. "Biological and Synthetic Scaffold: An Extra Cellular Matrix for Constructive Tissue Engineering," International Journal of Medical Research \& Review. 2016; 04 (10): 1882-1896.

[17] Schiffman JD, Schauer CL. "A review: electrospinning of biopolymer nanofibers and their applications," Polym. Rev. 2008; 48: 317-352.

[18] Zhang Y, Venugopal J, Huang Z-M, Lim CT, Ramakrishna S. "Crosslinking of the electrospun gelatin nanofibers," Polymer. 2006; 47: 2911-2917. 
[19] Chen C, Chuanbao C, Xilan M, Tang Y. "Preparation of nonwoven mats from all-aqueous silk fibroin solution with electrospinning method," Polymer 2006; 47: 6322-6327.

[20] Ghasemi-Mobarakeh L, Prabhakaran MP, Morshed M, NasrEsfahani MH, Ramakrishna S. "Electrospun poly ( $\varepsilon-$ caprolactone)/ gelatin nanofibrous scaffolds for nerve tissue engineering," Biomaterials. 2008; 29: 4532-4539.

[21] Chong E, Phan T, Lim I, Zhang YZ, Bay BH, Ramakrishna S, Lim CT. "Evaluation of electrospun PCL/gelatin nanofibrous scaffold for wound healing and layered dermal reconstitution," Acta Biomater 2007; 3: 321-330.

[22] Ding D, Shyu W, Lin S. "Mesenchymal stem cells," Cell Transpl 2011; 20: 5-14.

[23] Ranjbaran H, Abediankenari S, Mohammadi M, Jafari N, Khalilian A, Rahmani Z, Momeninezhad-Amiri M, Ebrahimi P. "Wharton's Jelly Derived-Mesenchymal Stem Cells: Isolation and Characterization," Acta Med Iran 2018; 56 (1): 28-33.

[24] Jaianand K, Balaji P. "Clinical Prospects of Scale-Up foetal Wharton's Jelly-Derived Multipotent Stromal Cells to fulfil the Therapeutic Demands," Int J Pharm. Bio. Sci. 2015; 6 (4): B882-B894. s.

[25] Jaianand K, Balaji P. "Isolation, Characterization and ScaleUp of Foetal Amniotic Membrane-Derived Multipotent Stromal Cells for Therapeutic Applications," Int J Pharm. Bio. Sci. 2015; 6 (2): 376-385.

[26] Dominici M, Le BK, Mueller I, Slaper-Cortenbach I, Marini F, Krause D, Deans R, Keating A, Prockop DJ, Horwitz E. "Minimal criteria for defining multipotent mesenchymal stromal cells. The International Society for Cellular Therapy position statement," Cytotherapy. 2006; 8 (4): 315-317.

[27] Sutthiphong S, Pavasant P, Supaphol P. "Electrospun 1, 6diisocyanatohexane-extended poly (1, 4-butylene succinate) fiber mats and their potential for use as bone scaffolds," Polymer. 2009; 50: 1548-1558.

[28] Jain KG, Mohanty S, Ray AR, Malhotra R, Airan B. "Culture $\&$ differentiation of mesenchymal stem cell into osteoblast on degradable biomedical composite scaffold: In-vitro study," Indian J Med Res. 2015; 142 (6): 747-758.
[29] Jaianand K, Saurabh K, Suriya N, Firdosh M. "Dehydrated Amniotic Allograft seeded with Stem cells: The Clinical Perspectives for Wound healing," J Stem Cell Regen Biol. 2019; JSRB-19-RA-2954.

[30] Al-Nasiry S, Geusens N, Hanssens M, Luyten C, Pijnenborg R. "The use of Alamar Blue assay for quantitative analysis of viability, migration and invasion of choriocarcinoma cells," Hum Reprod. 2007; 22 (5): 1304-1309.

[31] Gnana R, Girija K and Ravichandran N. "In-vitro studies of anti-inflammatory and antioxidant activity of 4-(3H) quinazolinone derivatives," Rasayan journal. 2011; (2): 418424.

[32] Stocum DL, Zupanc GKH. "Stretching the limits: Stem cells in regeneration science," Developmental Dynamics. 2008; 237: 3648-71.

[33] Chamberlain G, Fox J, Ashton B, Middleton J. "Concise review: Mesenchymal stem cells: Their phenotype, differentiation capacity, immunological features, and potential for homing," Stem Cells. 2007; 25: 2739-49.

[34] Sarah S and David LK. "Stem cell- and scaffold-based tissue engineering approaches to osteochondral regenerative medicine," Semin Cell Dev Biol. 2009; 20 (6): 646-655.

[35] Amariei N, Manea LR, Bertea AP, Bertea A, Popa A. "The influence of polymer solution on the properties of electrospun 3D nanostructures," IOP conf.ser. Mater. sci eng. 2017; 209 (1): 012092.

[36] Ruijuan $\mathrm{Y}$, Jing $\mathrm{H}$, Guolong $\mathrm{M}$, Bo Jiang and Fang Wu, "Electrospun PCL/Gelatin composite fibrous scaffolds: mechanical properties and cellular responses," Journal of Biomaterials Science. 2016; Polymer Edition, DOI: 10.1080/09205063.2016.1160560.

[37] Pal J, Wu D, Hakkarainen M, Srivastava, RK. "The viscoelastic interaction between dispersed and continuous phase of PCL/HA-PVA oil-in-water emulsion uncovers the theoretical and experimental basis for fiber formation during emulsion electrospinning," eur. polym. J. 2017; 96: 44-54. 